\title{
IN VITRO SULFORHODAMINE B ASSAY EVALUATION OF NOVEL 2-PHENYL BENZOFURANONE DERIVATIVES ON HUMAN SKIN CANCER CELL LINE G361
}

\author{
1* Raghava Doonaboyina, ${ }^{2}$ Abhilasha Mittal, ${ }^{3}$ Sridhar Babu Gummadi \\ 1. Department of Pharmaceutical Chemistry, Institute of Pharmacy, NIMS University, Jaipur Rajasthan, India \\ 2. Department of Pharmaceutical Chemistry, Institute of Pharmacy, NIMS University, Jaipur Rajasthan, India \\ 3. Department of Pharmaceutical Chemistry, Shivani College of Pharmacy, Warangal, Telangana, India
}

\begin{abstract}
The newly synthesized compounds are being tested for in vitro anti-cancer activity. Method used for In Vitro testing is Sulforhodamine B assay also known SRB assay. Cell lines were prepared and homoginised and disassociated with the help of trypsin. Then trypsin was inactivated with fetal bovine serum. Then cell concentration was determined. Synthesised molecules where prepared in to four different dillutions and exposed to cell lines. The procedure was also compared with standard drug doxorubicin. All the cell medium was incubated 37 degrees centigrade in a humidified incubator with 5 percentage CO. The plates were stainned and fixed with trichloroacetic acid. Finally the plates were incubated in orbital shaker incubater and absorbance was measured in micro plate reader at 510nm. All compounds (1-30) showed similar anticancer activity of compounds (IA, IB, ID,IE,IF, IIB,IIC, IIIA, IVB, IVF, VA, VC, VD, VE.) were more potent when compared to the rest of the compounds synthesized.
\end{abstract}

Keywords: Sulforhodamine B assay, trypsin, fetal bovine serum, doxorubicin, micro plate reader.

Article Info: Received 10 July 2019; $\quad$ Review Completed 18 Aug 2019; Accepted 23 Aug 2019; Available online 30 Aug 2019

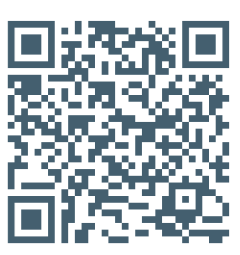

Cite this article as:

Doonaboyina R, Mittal A, Gummadi SB, IN VITRO SULFORHODAMINE B ASSAY EVALUATION OF NOVEL 2-PHENYL BENZOFURANONE DERIVATIVES ON HUMAN SKIN CANCER CELL LINE G361, Journal of Drug Delivery and Therapeutics. 2019; 9(4-A):385-389 http://dx.doi.org/10.22270/jddt.v9i4-A.3434

*Address for Correspondence:

Raghava Doonaboyina, Research Scholar, NIMS Institute of Pharmacy, NIMS University, Rajasthan

\section{INTRODUCTION}

Cancer is also called as malignancy disease where group of abnormal cells undergo cell division uncontrollable beyond regular methods. In broad term malignancy is an illness of cellular changes that results in uncontrolled cell division, sometimes it may be rapid cell division or cell grow or divide in a slow rate. Normal cell shall be divided by controlled signals which instruct cells to divide, differentiation or to die. Whereas malignant cells builds autonomy signals causes uncontrolled growth with proliferation. ${ }^{1-3}$ Sulfordamine B assay is an In Vivo model where an extrinsic method to estimate anti-tumour activity. ${ }^{4-5}$ it is the method which was developed in the year $1990 .^{3}$ This Method majorly depends on the stoichiometric binding of SRB dye with proteins at mild acidic conditions which can be extracted by reverse basic canditions.6-7 The process is majorly categorised into four stages preparation of treatment, incubation of cells with treatment of choice, cell fixation and SRB staining, and absorbance measurement. 8

\section{MATERIALS AND METHODS:}

96, 384 well clear flat-bottom polystyrene tissue-culture plates, 96 well PCR plates, $100 \mathrm{~mm}$ tissue culture plates, $15 \mathrm{ml}$ falcon tubes, $1.5 \mathrm{ml}$ eppendorf tubes, pipette tips, 125 microliter and 1250 microliter matrix pipette, human skin cancer cell line G361, culture medium, sterile and nonsterile reservoir, opti-MEM or serum free medium, phosphate buffer saline (PBS),2.5\%w/v trypsin solution, fatal bovine serum, tryptan blue, trichloroacetic acid, sulforhodamine B sodium salt in $1 \% \mathrm{v} / \mathrm{v}$ acetic acid, acetic acid, $10 \mathrm{mM}$ unbuffered tris base solution, Lipofectamine RNA imax, mirvana miRNA mimics, mi RNA precursor molecules - negative control. ${ }^{9}$

Testing method: Sulforhodamine B assay.

\section{Sample solution preparation:}

Synthesized compounds were prepared by in the concentration of 50 microliter and other dilution and added 96-well plates at a triplicate final volume was made up to 100 microliter with DMSO. 
Table 01 List of synthesized molecules and their concentration

\begin{tabular}{|c|c|c|c|c|c|}
\hline S.NO & SAMPLE ID & DILUTION-1 & DILUTION-2 & DILUTION-3 & DILUTION-4 \\
\hline 1 & PG-1A & $10^{-7}$ & $10^{-6}$ & $10^{-5}$ & $10^{-4}$ \\
\hline 2 & PG-1B & $10^{-7}$ & $10^{-6}$ & $10^{-5}$ & $10^{-4}$ \\
\hline 3 & PG-1C & $10^{-7}$ & $10^{-6}$ & $10^{-5}$ & $10^{-4}$ \\
\hline 4 & PG-1D & $10^{-7}$ & $10^{-6}$ & $10^{-5}$ & $10^{-4}$ \\
\hline 5 & PG-1E & $10^{-7}$ & $10^{-6}$ & $10^{-5}$ & $10^{-4}$ \\
\hline 6 & PG-1F & $10^{-7}$ & $10^{-6}$ & $10^{-5}$ & $10^{-4}$ \\
\hline 7 & RS-1A & $10^{-7}$ & $10^{-6}$ & $10^{-5}$ & $10^{-4}$ \\
\hline 8 & RS-1B & $10^{-7}$ & $10^{-6}$ & $10^{-5}$ & $10^{-4}$ \\
\hline 9 & RS-1C & $10^{-7}$ & $10^{-6}$ & $10^{-5}$ & $10^{-4}$ \\
\hline 10 & RS -1D & $10^{-7}$ & $10^{-6}$ & $10^{-5}$ & $10^{-4}$ \\
\hline 11 & RS -1E & $10^{-7}$ & $10^{-6}$ & $10^{-5}$ & $10^{-4}$ \\
\hline 12 & $\mathrm{RS}-1 \mathrm{~F}$ & $10^{-7}$ & $10^{-6}$ & $10^{-5}$ & $10^{-4}$ \\
\hline 13 & OR-1A & $10^{-7}$ & $10^{-6}$ & $10^{-5}$ & $10^{-4}$ \\
\hline 14 & OR-1B & $10^{-7}$ & $10^{-6}$ & $10^{-5}$ & $10^{-4}$ \\
\hline 15 & OR-1C & $10^{-7}$ & $10^{-6}$ & $10^{-5}$ & $10^{-4}$ \\
\hline 16 & OR-1D & $10^{-7}$ & $10^{-6}$ & $10^{-5}$ & $10^{-4}$ \\
\hline 17 & OR -1E & $10^{-7}$ & $10^{-6}$ & $10^{-5}$ & $10^{-4}$ \\
\hline 18 & OR $-1 \mathrm{~F}$ & $10^{-7}$ & $10^{-6}$ & $10^{-5}$ & $10^{-4}$ \\
\hline 19 & HQ-1A & $10^{-7}$ & $10^{-6}$ & $10^{-5}$ & $10^{-4}$ \\
\hline 20 & HQ-1B & $10^{-7}$ & $10^{-6}$ & $10^{-5}$ & $10^{-4}$ \\
\hline 21 & HQ-1C & $10^{-7}$ & $10^{-6}$ & $10^{-5}$ & $10^{-4}$ \\
\hline 22 & HQ-1D & $10^{-7}$ & $10^{-6}$ & $10^{-5}$ & $10^{-4}$ \\
\hline 23 & HQ -1E & $10^{-7}$ & $10^{-6}$ & $10^{-5}$ & $10^{-4}$ \\
\hline 24 & HQ -1F & $10^{-7}$ & $10^{-6}$ & $10^{-5}$ & $10^{-4}$ \\
\hline 25 & HL-1A & $10^{-7}$ & $10^{-6}$ & $10^{-5}$ & $10^{-4}$ \\
\hline 26 & HL-1B & $10^{-7}$ & $10^{-6}$ & $10^{-5}$ & $10^{-4}$ \\
\hline 27 & HL-1C & $10^{-7}$ & $10^{-6}$ & $10^{-5}$ & $10^{-4}$ \\
\hline 28 & HL-1D & $10^{-7}$ & $10^{-6}$ & $10^{-5}$ & $10^{-4}$ \\
\hline 29 & HL -1E & $10^{-7}$ & $10^{-6}$ & $10^{-5}$ & $10^{-4}$ \\
\hline 30 & HL -1F & $10^{-7}$ & $10^{-6}$ & $10^{-5}$ & $10^{-4}$ \\
\hline 31 & ADR & $10^{-7}$ & $10^{-6}$ & $10^{-5}$ & $10^{-4}$ \\
\hline
\end{tabular}

\section{Cell line preparation:}

Trypsinization: Cell line monolayer's are washed with sterilized phosphate buffer saline (PBS). PBS was removed from the medium and then added $1 \mathrm{ml}$ of $0.25 \%(\mathrm{w} / \mathrm{v})$ trypsin to evenly cover the cell line surface. Then incubate the cell medium at $37^{\circ} \mathrm{C}$ for 5 min until cells start to dissociate from its surface. Trypsin was inactivated with 10 volumes of culture medium containing (fetal bovine serum) FBS by mixing up and down until single homogenous single cell suspension. Single cell suspension was transferred to a sterile falcon tube. ${ }^{10}$

\section{Determination of cell concentration.}

Single cell suspension was filled in a falcon tube and $0.4 \%$ (w/v) tryptan blue solution in a ratio $1: 1$ and then cell suspension was measured for cell concentration in hematocytometer chamber under a microscope to determine cell viability prior cell seeding and verified for cell health.

Cell concentration was adjusted with 10\% FBS growth medium in 96 well with cell density of 50 microliter. The cell suspension was stored in sterile reagent container for multichannel pipette.

\section{Treatment exposure:}

50 microliter of treatment sample was transferred in to each well of 96 well plate. Then cell suspension of 50 microliter was added to each well. Even cell distribution was observed at the bottom of the plate. A short spin was performed to the plate for 20 seconds. Three wells are incubated with above procedure with standard drug doxorubicin.

\section{Blank preparation:}

Three wells are filled with vehicle DMSO and cell suspension for untreated vehicle control, three wells are filled with DMSO only for background subtraction.

\section{Incubation:}

Plates are incubated at $37^{\circ} \mathrm{C}$ in a humidified incubator with $5 \%$ CO until plates are recorded.

\section{Cell fixation and staining:}

To the treated cell lines 25 microliter of cold trichloroacetic acid $50 \%(\mathrm{w} / \mathrm{v})$ is added to each well directly to medium supernatant, and plates are incubated at $4{ }^{\circ} \mathrm{C}$ for 1 hour. The plates are washed four times with submerging the plate in a tub with slow running water. Gently tapping was done to remove the excess water in to a paper towel and air dried at room temperature.

A 50 microliter $0.04 \%$ SRB solution was added to each well and incubated and kept at room temperature for 1 hour and quickly rinse the plates washed four times with $1 \%(\mathrm{v} / \mathrm{v})$ acetic acid (200microliter) to remove unbound dye. Then plates air dried at room temperature and verified for the presence of air bubbles.

Absorbance measurement: 
50-100 microliter of tris-base solution of PH 10.5 was added to each well and shakes the plate in an orbital shaker incubator for $10 \mathrm{~min}$ to solubilise the protein bound dye. Then absorbance was measured at $510 \mathrm{~nm}$ in a micro plate reader.

\section{Calculations:}

Background absorbance was removed from all vessels

Cell percentage growth $=$ Absorbance sample $/$ Absorbance negative control X 100
$\%$ Growth inhibition $=100-\%$ Cell growth.

Growth inhibition was calculated of $50 \%$ was calculated as GI 50 .

TGI: Drug concentration resulting in total growth inhibition (TGI).

$\mathrm{LC}_{50}$ : Concentration of drug resulting in a $50 \%$ reduction in the measured protein at the end of the drug treatment (concentration of drug causing lethality to $50 \%$ of the cells as compared to that at the beginning) indicating a net loss of cells following treatment.

Table 02 Experimental results of induvidual experiments

\begin{tabular}{|c|c|c|c|c|c|c|c|c|}
\hline & \multicolumn{4}{|c|}{ Experiment - 1} & \multicolumn{4}{|c|}{ Experiment - 2} \\
\hline SAMPLE ID & $10^{-7} M$ & $10^{-6} \mathrm{M}$ & $10^{-5} \mathrm{M}$ & $10^{-4} \mathrm{M}$ & $10^{-7} \mathrm{M}$ & $10^{-6} \mathrm{M}$ & $10^{-5} \mathrm{M}$ & $10^{-4} \mathrm{M}$ \\
\hline PG-1A & 100.0 & 100.0 & 100.0 & -61.5 & 100.0 & 100.0 & 79.8 & -57.0 \\
\hline PG-1B & 95.3 & 94.3 & 63.2 & 29.7 & 100.0 & 92.1 & 65.4 & 34.4 \\
\hline PG-1C & 100.0 & 100.0 & 97.3 & 46.6 & 100.0 & 100.0 & 77.2 & 39.5 \\
\hline PG-1D & 100.0 & 100.0 & 100.0 & -36.5 & 100.0 & 100.0 & 74.9 & -30.0 \\
\hline PG-1E & 100.0 & 100.0 & 100.0 & -61.5 & 100.0 & 100.0 & 79.8 & -57.0 \\
\hline PG-1F & 100.0 & 100.0 & 100.0 & -61.2 & 100.0 & 100.0 & 74.1 & -62.9 \\
\hline RS-1A & 100.0 & 100.0 & 100.0 & 21.9 & 100.0 & 100.0 & 89.5 & 19.9 \\
\hline RS-1B & 100.0 & 100.0 & 71.2 & 2.2 & 100.0 & 90.8 & 88.0 & 10.2 \\
\hline RS-1C & 100.0 & 100.0 & 100.0 & -20.8 & 100.0 & 100.0 & 100.0 & -40.9 \\
\hline RS -1D & 100.0 & 100.0 & 100.0 & 22.4 & 100.0 & 100.0 & 100.0 & 9.1 \\
\hline RS -1E & 100.0 & 100.0 & 100.0 & 52.5 & 100.0 & 100.0 & 100.0 & 41.6 \\
\hline RS -1F & 100.0 & 100.0 & 100.0 & 25.6 & 100.0 & 100.0 & 86.5 & 15.8 \\
\hline OR-1A & 100.0 & 100.0 & 100.0 & 3.5 & 100.0 & 72.4 & 12.7 & -11.2 \\
\hline OR-1B & 100.0 & 100.0 & 100.0 & 61.2 & 100.0 & 92.4 & 77.8 & 54.6 \\
\hline OR-1C & 100.0 & 100.0 & 100.0 & 80.9 & 100.0 & 100.0 & 99.2 & 63.7 \\
\hline OR-1D & 100.0 & 100.0 & 100.0 & 25.6 & 100.0 & 100.0 & 86.5 & 15.8 \\
\hline OR -1E & 100.0 & 100.0 & 100.0 & 70.9 & 100.0 & 100.0 & 100.0 & 61.8 \\
\hline OR -1F & 100.0 & 100.0 & 100.0 & 39.8 & 100.0 & 100.0 & 100.0 & 14.5 \\
\hline HQ-1A & 100.0 & 100.0 & 100.0 & 30.1 & 100.0 & 100.0 & 100.0 & 25.1 \\
\hline HQ-1B & 100.0 & 100.0 & 100.0 & -12.1 & 100.0 & 100.0 & 100.0 & -37.6 \\
\hline HQ-1C & 100.0 & 100.0 & 100.0 & 15.3 & 100.0 & 100.0 & 100.0 & 12.2 \\
\hline HQ-1D & 100.0 & 100.0 & 100.0 & 46.9 & 100.0 & 100.0 & 99.5 & 43.8 \\
\hline HQ -1E & 100.0 & 100.0 & 100.0 & 70.9 & 100.0 & 100.0 & 100.0 & 61.8 \\
\hline $\mathrm{HQ}-1 \mathrm{~F}$ & 100.0 & 100.0 & 100.0 & -12.1 & 100.0 & 100.0 & 100.0 & -37.6 \\
\hline HL-1A & 100.0 & 100.0 & 71.2 & 2.2 & 100.0 & 90.8 & 88.0 & 10.2 \\
\hline HL-1B & 100.0 & 100.0 & 100.0 & 21.9 & 100.0 & 100.0 & 89.5 & 19.9 \\
\hline HL-1C & 100.0 & 100.0 & 100.0 & -61.2 & 100.0 & 100.0 & 74.1 & -62.9 \\
\hline HL-1D & 100.0 & 100.0 & 100.0 & -61.5 & 100.0 & 100.0 & 79.8 & -57.0 \\
\hline HL -1E & 100.0 & 100.0 & 100.0 & -36.5 & 100.0 & 100.0 & 74.9 & -30.0 \\
\hline $\mathrm{HL}-1 \mathrm{~F}$ & 100.0 & 100.0 & 97.3 & 46.6 & 100.0 & 100.0 & 77.2 & 39.5 \\
\hline ADR & 48.3 & -44.4 & -41.2 & -55.0 & -53.9 & -65.2 & -69.4 & -73.5 \\
\hline
\end{tabular}

\begin{tabular}{|c|c|c|c|c|c|c|c|c|}
\hline & \multicolumn{4}{|c|}{ Experiment - 3 } & \multicolumn{4}{c|}{ Average Values } \\
\hline SAMPLE ID & $\mathbf{1 0}^{-7} \mathbf{M}$ & $\mathbf{1 0}^{-6} \mathbf{M}$ & $\mathbf{1 0}^{-5} \mathbf{M}$ & $\mathbf{1 0}^{-4} \mathbf{M}$ & $\mathbf{1 0}^{-7} \mathbf{M}$ & $\mathbf{1 0}^{-6} \mathbf{M}$ & $\mathbf{1 0}^{-5} \mathbf{M}$ & $\mathbf{1 0}^{-4} \mathbf{M}$ \\
\hline PG-1A & 100.0 & 89.6 & 79.1 & -64.4 & $\mathbf{1 0 0 . 0}$ & $\mathbf{9 6 . 5}$ & $\mathbf{8 6 . 3}$ & -61.0 \\
\hline PG-1B & 107.6 & 70.1 & 69.0 & 28.6 & $\mathbf{1 0 1 . 0}$ & $\mathbf{8 5 . 5}$ & $\mathbf{6 5 . 9}$ & $\mathbf{3 0 . 9}$ \\
\hline PG-1C & 100.0 & 89.3 & 75.3 & 34.6 & $\mathbf{1 0 0 . 0}$ & $\mathbf{9 6 . 4}$ & $\mathbf{8 3 . 3}$ & $\mathbf{4 0 . 0}$ \\
\hline PG-1D & 100.0 & 84.0 & 79.2 & -37.7 & $\mathbf{1 0 0 . 0}$ & $\mathbf{9 4 . 7}$ & $\mathbf{8 4 . 7}$ & $-\mathbf{3 4 . 7}$ \\
\hline PG-1E & 100.0 & 89.6 & 79.1 & -64.4 & $\mathbf{1 0 0 . 0}$ & $\mathbf{9 6 . 5}$ & $\mathbf{8 6 . 3}$ & $-\mathbf{6 1 . 0}$ \\
\hline PG-1F & 100.0 & 89.4 & 80.2 & -63.3 & $\mathbf{1 0 0 . 0}$ & $\mathbf{9 6 . 5}$ & $\mathbf{8 4 . 8}$ & $-\mathbf{6 2 . 5}$ \\
\hline RS-1A & 100.0 & 100.0 & 86.1 & 15.8 & $\mathbf{1 0 0 . 0}$ & $\mathbf{1 0 0 . 0}$ & $\mathbf{9 1 . 9}$ & $\mathbf{1 9 . 2}$ \\
\hline RS-1B & 100.0 & 86.7 & 73.6 & 8.5 & $\mathbf{1 0 0 . 0}$ & $\mathbf{9 2 . 5}$ & $\mathbf{7 7 . 6}$ & $\mathbf{6 . 9}$ \\
\hline RS-1C & 100.0 & 100.0 & 100.0 & -45.2 & $\mathbf{1 0 0 . 0}$ & $\mathbf{1 0 0 . 0}$ & $\mathbf{1 0 0 . 0}$ & $-\mathbf{3 5 . 6}$ \\
\hline RS -1D & 100.0 & 100.0 & 100.0 & 13.4 & $\mathbf{1 0 0 . 0}$ & $\mathbf{1 0 0 . 0}$ & $\mathbf{1 0 0 . 0}$ & $\mathbf{1 5 . 0}$ \\
\hline RS -1E & 100.0 & 100.0 & 100.0 & 42.2 & $\mathbf{1 0 0 . 0}$ & $\mathbf{1 0 0 . 0}$ & $\mathbf{1 0 0 . 0}$ & $\mathbf{4 5 . 4}$ \\
\hline RS -1F & 100.0 & 100.0 & 100.0 & 12.0 & $\mathbf{1 0 0 . 0}$ & $\mathbf{1 0 0 . 0}$ & $\mathbf{9 5 . 5}$ & $\mathbf{1 7 . 8}$ \\
\hline OR-1A & 100.0 & 100.0 & 17.5 & -5.8 & $\mathbf{1 0 0 . 0}$ & $\mathbf{9 0 . 8}$ & $\mathbf{4 3 . 4}$ & -4.5 \\
\hline OR-1B & 100.0 & 100.0 & 99.1 & 39.3 & $\mathbf{1 0 0 . 0}$ & $\mathbf{9 7 . 5}$ & $\mathbf{9 2 . 3}$ & $\mathbf{5 1 . 7}$ \\
\hline OR-1C & 100.0 & 100.0 & 100.0 & 49.8 & $\mathbf{1 0 0 . 0}$ & $\mathbf{1 0 0 . 0}$ & $\mathbf{9 9 . 7}$ & $\mathbf{6 4 . 8}$ \\
\hline
\end{tabular}




\begin{tabular}{|c|c|c|c|c|c|c|c|c|} 
OR-1D & 100.0 & 100.0 & 100.0 & 12.0 & $\mathbf{1 0 0 . 0}$ & $\mathbf{1 0 0 . 0}$ & $\mathbf{9 5 . 5}$ & $\mathbf{1 7 . 8}$ \\
\hline OR -1E & 100.0 & 100.0 & 100.0 & 51.8 & $\mathbf{1 0 0 . 0}$ & $\mathbf{1 0 0 . 0}$ & $\mathbf{1 0 0 . 0}$ & $\mathbf{6 1 . 5}$ \\
\hline OR -1F & 100.0 & 100.0 & 100.0 & 21.5 & $\mathbf{1 0 0 . 0}$ & $\mathbf{1 0 0 . 0}$ & $\mathbf{1 0 0 . 0}$ & $\mathbf{2 5 . 3}$ \\
\hline HQ-1A & 100.0 & 100.0 & 100.0 & 35.1 & $\mathbf{1 0 0 . 0}$ & $\mathbf{1 0 0 . 0}$ & $\mathbf{1 0 0 . 0}$ & $\mathbf{3 0 . 1}$ \\
\hline HQ-1B & 100.0 & 100.0 & 100.0 & -40.3 & $\mathbf{1 0 0 . 0}$ & $\mathbf{1 0 0 . 0}$ & $\mathbf{1 0 0 . 0}$ & $-\mathbf{3 0 . 0}$ \\
\hline HQ-1C & 100.0 & 100.0 & 100.0 & 13.1 & $\mathbf{1 0 0 . 0}$ & $\mathbf{1 0 0 . 0}$ & $\mathbf{1 0 0 . 0}$ & $\mathbf{1 3 . 5}$ \\
\hline HQ-1D & 100.0 & 100.0 & 100.0 & 41.0 & $\mathbf{1 0 0 . 0}$ & $\mathbf{1 0 0 . 0}$ & $\mathbf{9 9 . 8}$ & $\mathbf{4 3 . 9}$ \\
\hline HQ -1E & 100.0 & 100.0 & 100.0 & 51.8 & $\mathbf{1 0 0 . 0}$ & $\mathbf{1 0 0 . 0}$ & $\mathbf{1 0 0 . 0}$ & $\mathbf{6 1 . 5}$ \\
\hline HQ -1F & 100.0 & 100.0 & 100.0 & -40.3 & $\mathbf{1 0 0 . 0}$ & $\mathbf{1 0 0 . 0}$ & $\mathbf{1 0 0 . 0}$ & $\mathbf{- 3 0 . 0}$ \\
\hline HL-1A & 100.0 & 86.7 & 73.6 & 8.5 & $\mathbf{1 0 0 . 0}$ & $\mathbf{9 2 . 5}$ & $\mathbf{7 7 . 6}$ & $\mathbf{6 . 9}$ \\
\hline HL-1B & 100.0 & 100.0 & 86.1 & 15.8 & $\mathbf{1 0 0 . 0}$ & $\mathbf{1 0 0 . 0}$ & $\mathbf{9 1 . 9}$ & $\mathbf{1 9 . 2}$ \\
\hline HL-1C & 100.0 & 89.4 & 80.2 & -63.3 & $\mathbf{1 0 0 . 0}$ & $\mathbf{9 6 . 5}$ & $\mathbf{8 4 . 8}$ & $\mathbf{- 6 2 . 5}$ \\
\hline HL-1D & 100.0 & 89.6 & 79.1 & -64.4 & $\mathbf{1 0 0 . 0}$ & $\mathbf{9 6 . 5}$ & $\mathbf{8 6 . 3}$ & $\mathbf{- 6 1 . 0}$ \\
\hline HL -1E & 100.0 & 84.0 & 79.2 & -37.7 & $\mathbf{1 0 0 . 0}$ & $\mathbf{9 4 . 7}$ & $\mathbf{8 4 . 7}$ & $\mathbf{- 3 4 . 7}$ \\
\hline HL -1F & 100.0 & 89.3 & 75.3 & 34.0 & $\mathbf{1 0 0 . 0}$ & $\mathbf{9 6 . 4}$ & $\mathbf{8 3 . 3}$ & $\mathbf{4 0 . 0}$ \\
\hline ADR & -66.5 & -78.0 & -82.1 & -83.6 & $\mathbf{- 2 4 . 0}$ & $\mathbf{- 6 2 . 5}$ & $\mathbf{- 6 4 . 2}$ & $\mathbf{- 7 0 . 7}$ \\
\hline
\end{tabular}

Table 3 Anti-cancer activity with molar drug concentration

\begin{tabular}{|c|c|c|c|}
\hline \multirow[t]{2}{*}{ G361 } & \multicolumn{3}{|c|}{ Molar drug concentration } \\
\hline & LC 50 & TGI & GI50 \\
\hline PG-1A & $>10^{-4}$ & $3.2 * 10^{-5}$ & $2.02^{*} 10^{-6}$ \\
\hline PG-1B & $>10^{-4}$ & $>10^{-4}$ & $2.8^{*} 10^{-6}$ \\
\hline PG-1C & $>10^{-4}$ & $>10^{-4}$ & $3.8 * 10^{-5}$ \\
\hline PG-1D & $>10^{-4}$ & $3.7 * 10^{-5}$ & $2.2^{*} 10^{-6}$ \\
\hline$P G-1 E$ & $>10^{-4}$ & $3.2^{*} 10^{-5}$ & $2.04 * 10^{-6}$ \\
\hline PG-1F & $>10^{-4}$ & $3.2^{*} 10^{-5}$ & $2.02^{*} 10^{-6}$ \\
\hline RS-1A & $>10^{-4}$ & $>10^{-4}$ & $3.2^{*} 10^{-5}$ \\
\hline RS-1B & $>10^{-4}$ & $>10^{-4}$ & $2.6 * 10-6$ \\
\hline RS-1C & $>10^{-4}$ & $>10^{-4}$ & $2.3^{*} 10^{-6}$ \\
\hline RS -1D & $>10^{-4}$ & $>10^{-4}$ & $3.2^{*} 10^{-5}$ \\
\hline RS -1E & $>10^{-4}$ & $>10^{-4}$ & $>10^{-4}$ \\
\hline $\mathrm{RS}-1 \mathrm{~F}$ & $>10^{-4}$ & $>10^{-4}$ & $3.2^{*} 10^{-5}$ \\
\hline OR-1A & $>10^{-4}$ & $3.7 * 10^{-5}$ & $2.1 * 10^{-6}$ \\
\hline OR-1B & $>10^{-4}$ & $>10^{-4}$ & $>10^{-4}$ \\
\hline OR-1C & $>10^{-4}$ & $>10^{-4}$ & $>10^{-4}$ \\
\hline OR-1D & $>10^{-4}$ & $>10^{-4}$ & $3.2^{*} 10^{-5}$ \\
\hline OR-1E & $>10^{-4}$ & $>10^{-4}$ & $>10^{-4}$ \\
\hline OR -1F & $>10^{-4}$ & $>10^{-4}$ & $3.6 * 10^{-5}$ \\
\hline HQ-1A & $>10^{-4}$ & $>10^{-4}$ & $3.9 * 10^{-5}$ \\
\hline HQ-1B & $>10^{-4}$ & $>10^{-4}$ & $2.4^{*} 10^{-6}$ \\
\hline HQ-1C & $>10^{-4}$ & $>10^{-4}$ & $3.2 * 10^{-5}$ \\
\hline HQ-1D & $>10^{-4}$ & $>10^{-4}$ & $>10^{-4}$ \\
\hline $\mathrm{HQ}-1 \mathrm{E}$ & $>10^{-4}$ & $>10^{-4}$ & $>10^{-4}$ \\
\hline HQ -1F & $>10^{-4}$ & $>10^{-4}$ & $2.4^{*} 10^{-6}$ \\
\hline HL-1A & $>10^{-4}$ & $>10^{-4}$ & $2.6 * 10^{-6}$ \\
\hline HL-1B & $>10^{-4}$ & $>10^{-4}$ & $3.2^{*} 10^{-5}$ \\
\hline HL-1C & $>10^{-4}$ & $3.2^{*} 10^{-5}$ & $2.02^{*} 10^{-6}$ \\
\hline HL-1D & $>10^{-4}$ & $3.2 * 10^{-5}$ & $2.04^{*} 10^{-6}$ \\
\hline HL -1E & $>10^{-4}$ & $3.7^{*} 10^{-5}$ & $2.2^{*} 10^{-6}$ \\
\hline HL -1F & $>10^{-4}$ & $>10^{-4}$ & $3.8 * 10^{-5}$ \\
\hline ADR & $1.7 * 10^{-7}$ & $<10^{-7}$ & $<10^{-7}$ \\
\hline
\end{tabular}


Fig 1 Anti-cancer results group-I

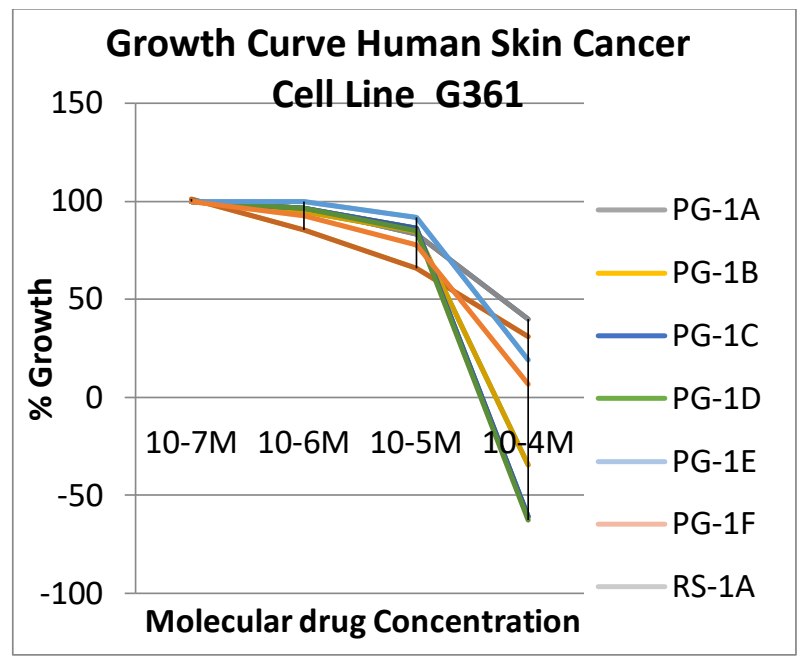

Fig 2 Anti-cancer results group-II

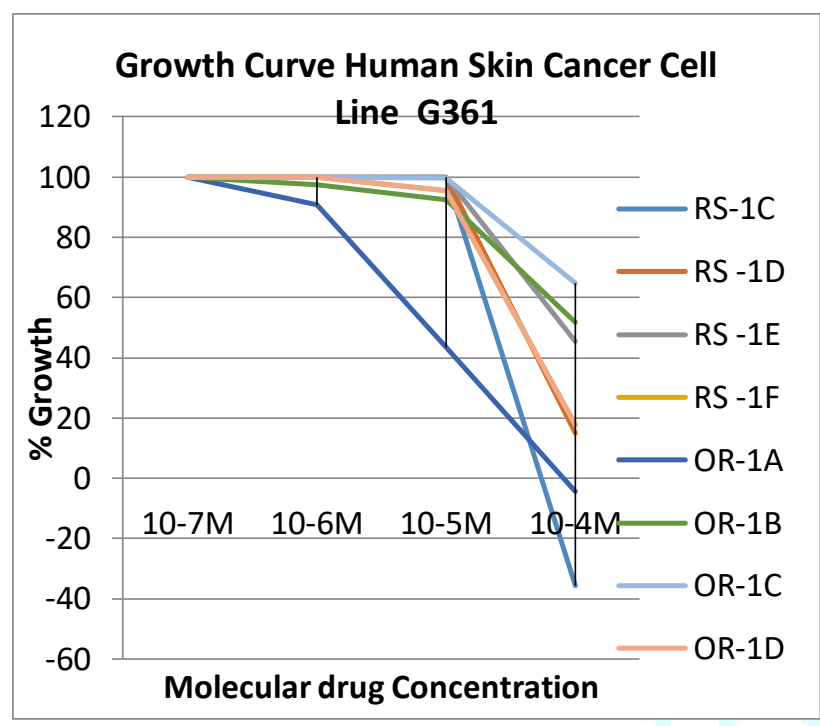

Fig 3 Anti-cancer results group-III

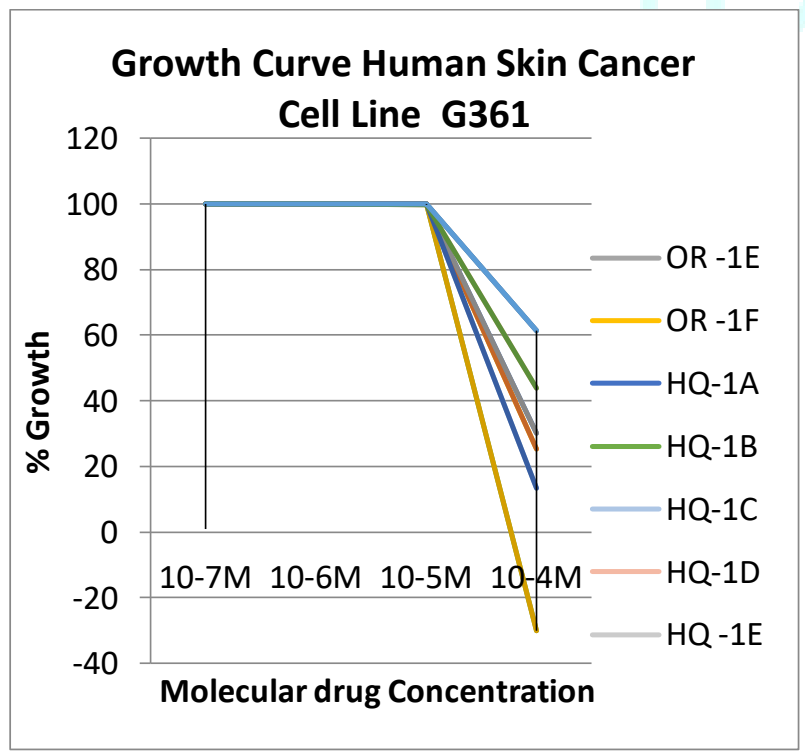

Fig 4 Anti cancer results group-IV

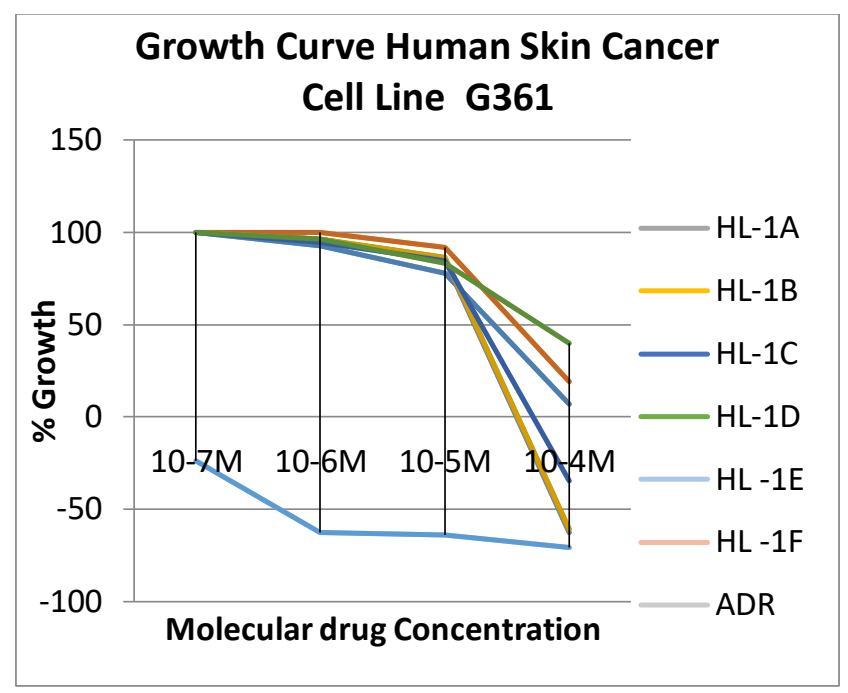

\section{CONCLUSION}

The synthesized molecules had been diluted at four different concentrations and Sulfordamine B assay method was opted for screening of anti-cancer activity. The results were compared with doxorubicin as standard drug at similar concentrations. The compounds exhibited good results.

\section{REFERENCES}

1. M. Mubeen and S. G. Kini, A review on the design and development of EGFR tyrosine kinase inhibitors in cancer therapy, International Journal of Therapeutic Applications, 2012; 5: 29-37.

2. Kasinski, A. L., Kelnar, K., Stahlhut, C., Orellana, E., Zhao, J. Shimer, E., Dysart, S., Chen, X.,Bader, A. G. and Slack, F. J. A combinatorial microRNA therapeutics approach to suppressing non-small cell lung cancer. Oncogene, 2015; 34(27): 3547-3555.

3. Skehan, P., Storeng, R., Scudiero, D., Monks, A., McMahon, J., Vistica, D., Warren, J. T.,

Bokesch, H., Kenney, S. and Boyd, M. R. New colorimetric cytotoxicity assay for anticancer-drug screening. J Natl Cancer Inst, 1990; 82(13): 1107-1112.

4. Vichai, V. and Kirtikara, K. Sulforhodamine B colorimetric assay for cytotoxicity screening. Nat Protoc, 2006; 1(3): 11121116.

5. A. Jemal, F. Bray, M. M. Center, J. Ferlay, E. Ward, and D. Forman, Global cancer statistics, CA Cancer Journal for Clinicians, 2011; 61(2): 69-90.

6. Venkatrao Adavirao, Gummadi Sridhar Babu, Medaboyina Padmavathi, Bobbala Ravi Kumar, Robert Michael Alex, Wolfgang Schuehly, Nebojsa Simic, Doris Kuhnelt, Achanta Venkata Narasimha Appa Rao. Novel steroidal glycosides from two Indian Caralluma species, C.stalagmifera \& C.indica. Olaf Kunert, Belvotagi Helvetica Chimica Acta, 2006; 89(2): 201209.

7. C. J. Murray and A. Lopez, The Global Burden of Disease, Harvard University Press, Cambridge, UK, 1996; 1(3): 11071112.

8. United Nations Development Programme (UNDP), Human Development Report Oxford University Press, Oxford, UK, 2000; 82(13): 69-90.

9. Kommidi Devender Reddy, Belvotagi VenkatRao Adavi Rao, Gummadi Sridhar Babu, Bobbala Ravi Kumar, Alessandra Braca, Antonio Vassalo, Nunziatina De Tommasi, Ghankota Venkateshwar Rao, Achanta Venkata Narasimha Appa Rao,Minor Pregnanes from Caralluma adscendens var. gracilis and Caralluma pauciflora; Fitoterapia, 2011; 82: 1039-1043.

10. Schwartsmann G, M. J. Ratain, G. M. Cragg et al., Anticancer drug discovery and development throughout the world," Journal of Clinical Oncology, 2002; 20(18): 47-59. 